PROCEEDINGS OF THE

AMERICAN MATHEMATICAL SOCIETY

Volume 132, Number 4, Pages 1211-1217

S 0002-9939(03)07270-8

Article electronically published on October 29, 2003

\title{
DENDRITES AND LIGHT MAPPINGS
}

\author{
JANUSZ J. CHARATONIK AND PAWE€ KRUPSKI
}

(Communicated by Alan Dow)

\begin{abstract}
It is shown that a metric continuum $X$ is a dendrite if and only if for every compact space (continuum) $Y$ and for every light confluent mapping $f: Y \rightarrow f(Y)$ such that $X \subset f(Y)$ there is a copy $X^{\prime}$ of $X$ in $Y$ for which the restriction $f \mid X^{\prime}: X^{\prime} \rightarrow X$ is a homeomorphism. As a corollary it follows that only dendrites have the lifting property with respect to light confluent mappings. Other classes of mappings $f$ are also discussed. This is a continuation of a previous study by the authors (2000), where open mappings $f$ were considered.
\end{abstract}

A well-known theorem of G. T. Whyburn (see [15, Theorem 2.4, p. 188]) says that if $f: X \rightarrow Y$ is a light open mapping from a compact space $X$ onto $Y$, and a dendrite $D$ is contained in $Y$, then for each point $x_{0} \in f^{-1}(D)$ there is a dendrite $D^{\prime} \subset X$ with $x_{0} \in D^{\prime}$ such that $f$ maps $D^{\prime}$ homeomorphically onto $D$. This result has been extended in several ways (see e.g. [12, [13] and [6]). It is shown in [1] that the property considered in Whyburn's theorem characterizes dendrites among all continua. In the present paper the characterization is further generalized. The most important characterization relies upon replacing open mappings by less restrictive ones such as confluent, locally confluent and some other light mappings.

All spaces considered in this paper are assumed to be metric, and all mappings are continuous. A continuum means a compact connected space. By a dendrite is meant a locally connected continuum containing no simple closed curve. We will also use the concepts of the following classes of mappings: light (i.e., 0-dimensional), monotone, open (denoted here by $\mathcal{O}$ ), OM-mappings (denoted by $\mathcal{O} \mathcal{M}$ ), confluent (denoted by $\mathcal{C}$ ), locally confluent (denoted by $\mathcal{L C}$ ), semi-confluent, and confluent over locally connected continua (denoted by $\mathcal{C}_{l c}$ ) as defined, e.g., in 6], 9] and [15].

Consider the following two conditions regarding a continuum $X$ and a class $\mathcal{M}$ of light mappings.

$\left(\omega_{0}(\mathcal{M})\right)$ For every compact space $Y$, for every light mapping $f: Y \rightarrow f(Y)$ in $\mathcal{M}$ with $X \subset f(Y)$ and for every point $y_{0} \in f^{-1}(X) \subset Y$ there exists a homeomorphic copy $X^{\prime}$ of $X$ in $Y$ with $y_{0} \in X^{\prime}$ such that the restriction $f \mid X^{\prime}: X^{\prime} \rightarrow f\left(X^{\prime}\right)=X$ is a homeomorphism.

$(\omega(\mathcal{M}))$ For every compact space $Y$ and for every light mapping $f: Y \rightarrow f(Y)$ in $\mathcal{M}$ with $X \subset f(Y)$ there exists a homeomorphic copy $X^{\prime}$ of $X$ in $Y$ such that the restriction $f \mid X^{\prime}: X^{\prime} \rightarrow f\left(X^{\prime}\right)=X$ is a homeomorphism.

Received by the editors March 14, 2001 and, in revised form, February 4, 2002.

2000 Mathematics Subject Classification. Primary 54C60, 54C65, 54E40, 54F50.

Key words and phrases. Confluent, continuum, dendrite, lifting, light, mapping, open.

(C)2003 American Mathematical Society 
Note that if $\mathcal{O}$ stands for the class of open mappings, then $\left(\omega_{0}(\mathcal{O})\right)$ and $(\omega(\mathcal{O}))$ coincide with conditions $\left(\omega_{0}\right)$ and $(\omega)$ from [1], respectively. It is proved in [1] that $\left(\omega_{0}\right)$ and $(\omega)$ are equivalent to the condition

$(\delta) X$ is a dendrite.

One can also consider conditions $\left(\gamma_{0}\right)$ and $(\gamma)$ obtained from $\left(\omega_{0}\right)$ and $(\omega)$, respectively, by replacing the phrase "every compact space $Y$ " with "every continuum $Y$ ". Note that the proof of the implication $(\gamma) \Longrightarrow(\delta)$ is exactly the same as the proof of the implication $(\omega) \Longrightarrow(\delta)$ in [1, Theorem 9, p. 1842] because the compact spaces $Y$ constructed in the proofs of Propositions 6 and 7 of [1] are continua, and the proof of Proposition 8 in [1] remains valid when $(\omega)$ is replaced by $(\gamma)$. Therefore, the following statement completes [1, Corollary 10, p. 1842].

1. Statement. For each continuum $X$, conditions $(\delta),\left(\omega_{0}\right),(\omega),\left(\gamma_{0}\right)$ and $(\gamma)$ are equivalent.

One can pose the following problem.

2. Problem. Does the equivalence in Statement 1 remain true if we replace openness of the light mapping $f$ by a less restrictive condition? In other words, for what (larger) classes $\mathcal{M}$ of light mappings are conditions $(\delta),\left(\omega_{0}\right),(\omega),\left(\gamma_{0}\right)$ and $(\gamma)$ equivalent?

In this paper we show the equivalence for the classes of OM-mappings, confluent, locally confluent and mappings confluent over locally connected continua.

3. Observation. Let $X$ be a continuum, and let $\mathcal{M}$ be a class of mappings between compact spaces. Then the following implications are obvious.

$(3.1)\left(\omega_{0}(\mathcal{M})\right) \Longrightarrow(\omega(\mathcal{M}))$.

(3.2) If $\mathcal{M}_{1} \subset \mathcal{M}_{2}$, then $\left(\omega_{0}\left(\mathcal{M}_{2}\right)\right) \Longrightarrow\left(\omega_{0}\left(\mathcal{M}_{1}\right)\right)$ and $\left(\omega\left(\mathcal{M}_{2}\right)\right) \Longrightarrow$ $\left(\omega\left(\mathcal{M}_{1}\right)\right)$.

In particular,

(3.3) if the class $\mathcal{M}$ contains the class $\mathcal{O}$ of open mappings, then

$$
\left(\omega_{0}(\mathcal{M})\right) \Longrightarrow\left(\omega_{0}\right) \text { and }(\omega(\mathcal{M})) \Longrightarrow(\omega) .
$$

In the light of Statement 1 and Observation 3 we get the next observation.

4. Observation. If the class $\mathcal{M}$ contains the class $\mathcal{O}$ of open mappings, and if, for a continuum $X$, implication $(\delta) \Longrightarrow\left(\omega_{0}(\mathcal{M})\right)$ holds, then all the conditions $(\delta),\left(\omega_{0}\right),(\omega),\left(\omega_{0}(\mathcal{M})\right)$ and $(\omega(\mathcal{M}))$ are equivalent.

According to Observation 4, Problem 2 reduces to the following question.

5. Question. For what classes $\mathcal{M}$ of mappings containing the class $\mathcal{O}$ does each dendrite $X$ satisfy condition $\left(\omega_{0}(\mathcal{M})\right)$ (i.e., does the implication $(\delta) \Longrightarrow\left(\omega_{0}(\mathcal{M})\right)$ hold)?

Recall that for $\mathcal{M}=\mathcal{O}$ the above-mentioned implication,

$$
(\delta) \Longrightarrow\left(\omega_{0}\right) \text {, }
$$

is just the quoted Whyburn's result; see [15, Theorem 2.4, p. 188]. Both assumptions regarding $f$ in $\left(\omega_{0}\right)$, i.e., lightness and openness, are essential in (6). For lightness, see e.g. [2, Example 7.1 p. 29]; for openness, one can easily construct a needed mapping from the unit interval onto a simple triod. 
7. Remark. A space is said to be locally dendritic provided that each of its points has a neighborhood that is a dendrite. If a metric space $Y$ is locally dendritic, $f: Y \rightarrow f(Y)$ is open and $f(Y)$ has no isolated point, then $f$ is light [3, Theorem 5 , p. 214]. Then lightness of $f$ can be omitted in conditions $\left(\omega_{0}\right)$ and $(\omega)$ (thus in implication (6) as well).

To show the next results, recall two definitions. A mapping $f: X \rightarrow Y$ between compact spaces is said to be:

- locally confluent provided that for each point $y \in Y$ there exists a closed neighborhood $V$ of $y$ in $Y$ such that $f \mid f^{-1}(V)$ is a confluent mapping of $f^{-1}(V)$ onto $V$ (see [4, p. 239] and [8, p. 106], and compare [9, Theorem 4.24, p. 19]);

- confluent over a continuum $B \subset Y$ provided that each component of $f^{-1}(B)$ is mapped under $f$ onto $B$ (see [6, p. 357] and compare the concept of a $C$-set in [5. p. 83, and Theorem 1, p. 84]).

8. Proposition. Each locally confluent mapping between compact spaces is confluent over locally connected continua.

Proof. Let $f: X \rightarrow Y$ be a locally confluent mapping between compact spaces, and let $B$ be a locally connected subcontinuum of $Y$. Then there is a finite family $\mathbf{V}$ of closed neighborhoods $V_{1}, \ldots, V_{j}$ of some points in $Y$ such that $\mathbf{V}$ covers $B$ and $f \mid f^{-1}\left(V_{i}\right)$ is confluent for each $i \in\{1, \ldots, j\}$. If $\eta$ is a Lebesgue number for this cover, then there is a finite family $\mathbf{B}$ of subcontinua $B_{1}, \ldots, B_{k}$ of $B$ such that $\operatorname{diam} B_{i}<\eta$ for each $i \in\{1, \ldots, k\}$ and $B=\bigcup\left\{B_{i}: i \in\{1, \ldots, k\}\right\}$, see e.g. [7, $\S 50$, II, Theorem 2, p. 256]. Let $C$ be a component of $f^{-1}(B)$. To show that $f(C)=B$ choose a point $c \in C$ and let $x \in B$. Then there is a chain $B_{i_{1}}, \ldots, B_{i_{m}}$ such that $f(c) \in B_{i_{1}}$ and $x \in B_{i_{m}}$. Since each member of $\mathbf{B}$ is contained in some member of $\mathbf{V}$, it follows that for each $i \in\left\{i_{1}, \ldots, i_{m}\right\}$ each component of $f^{-1}\left(B_{i}\right)$ is mapped onto $B_{i}$ under $f$. Consequently, there is in $C$ a chain $C_{1}, \ldots, C_{n}$ of these components such that $c \in C_{1}$ and $x \in f\left(C_{n}\right)$. Since $C_{n} \subset C$, we get $x \in f(C)$, as needed.

9. Remark. The implication in Proposition 8 cannot be reversed. Indeed, to see a mapping in $\mathcal{C}_{l c} \backslash \mathcal{L C}$, recall that a continuum $Y$ is said to be locally indecomposable provided that there exists $\varepsilon>0$ such that every subcontinuum of $Y$ of diameter less than $\varepsilon$ is indecomposable; and a continuum $Y$ is locally indecomposable if and only if each mapping from any continuum onto $Y$ is locally confluent (see [14] Theorem 10]). Let $Y$ be a hereditarily decomposable continuum containing no locally connected subcontinuum (see e.g. the continuum $\Sigma$ in [10, (2.8) and (2.9), pp. 14-16]). Then there are a continuum $X$ and a surjection $f: X \rightarrow Y$ such that $f$ is not locally confluent. Obviously $f \in \mathcal{C}_{l c}$, since the definition is satisfied vacuously.

10. Proposition. If a mapping $f$ between compact spaces is confluent over locally connected continua and the range space of $f$ is locally connected, then $f$ is confluent.

Proof. Let spaces $X$ and $Y$ be compact, $Y$ be locally connected, and let $f: X \rightarrow Y$ be in $\mathcal{C}_{l c}$. Take a subcontinuum $B$ of $Y$. Then there is a decreasing sequence of locally connected subcontinua $B_{n}$ of $Y$ such that $B=\bigcap\left\{B_{n}: n \in \mathbb{N}\right\}$ (see [7, $\S 50$, III, Theorem 1, p. 260]). Let $C$ be a component of $f^{-1}(B)$. For each $n \in \mathbb{N}$, let $C_{n}$ denote the component of $f^{-1}\left(B_{n}\right)$ with $C \subset C_{n}$. Since the continua $B_{n}$ are locally connected and $f \in \mathcal{C}_{l c}$, we have $f\left(C_{n}\right)=B_{n}$ for each $n$. The components 
$C_{n}$ form a decreasing sequence, too, and putting $C^{\prime}=\bigcap\left\{C_{n}: n \in \mathbb{N}\right\}$, it follows that $C \subset C^{\prime}$. On the other hand, $f\left(C^{\prime}\right)=f\left(\bigcap\left\{C_{n}: n \in \mathbb{N}\right\}\right)=\bigcap\left\{f\left(C_{n}\right): n \in\right.$ $\mathbb{N}\}=\bigcap\left\{B_{n}: n \in \mathbb{N}\right\}=B$, whence $C^{\prime} \subset f^{-1}(B)$, and therefore $C^{\prime}=C$. Thus $f$ is confluent, and the proof is finished.

The results of Chapter 4 of [9] summarized in [9, Table II, p. 28], Proposition 8 and Remark 9 imply the following inclusions, no one of which can be reversed:

$$
\mathcal{O} \subset \mathcal{O} \mathcal{M} \subset \mathcal{C} \subset \mathcal{L C} \subset \mathcal{C}_{l c}
$$

12. Proposition. Let a light surjective mapping $f$ be defined on a compact metric space. If either the domain or the range space of $f$ is locally connected, then the following conditions are equivalent:

(12.1) $f$ is open,

(12.2) $f$ is an OM-mapping,

(12.3) $f$ is confluent,

(12.4) $f$ is locally confluent,

(12.5) $f$ is confluent over locally connected continua.

Proof. The equivalence of conditions (12.1)-(12.4) is known; see [3, Proposition 1, p. 212] and [12, Proposition 2, p. 39].

Implications $(12.4) \Longrightarrow(12.5) \Longrightarrow(12.3)$ are stated in Propositions 8 and 10, respectively. Thus the proof is complete.

We will use Proposition 12 to extend Statement 1 from an open mapping $f$ to any mapping in (11). Namely, we have the following result.

13. Proposition. Let $\mathcal{M}$ denote any class of mappings listed in (11). Then for each continuum $X$, conditions $(\delta),\left(\omega_{0}(\mathcal{M})\right)$ and $(\omega(\mathcal{M}))$ are equivalent.

Proof. By (11) the class $\mathcal{M}$ contains the class of open mappings. Thus, according to Observation 4, to prove the equivalence it is enough to show the implication $(\delta) \Longrightarrow\left(\omega_{0}(\mathcal{M})\right)$.

So, let $Y$ be a compact space, $f: Y \rightarrow f(Y)$ be a light mapping with $f \in \mathcal{M}$, and let a dendrite $X \subset f(Y)$ and a point $y_{0} \in f^{-1}(X)$ be given. By (11) each mapping in $\mathcal{M}$ is in $\mathcal{C}_{l c}$, and so is $f$. Consider the following diagram, in which the horizontal arrows denote the inclusion mappings.

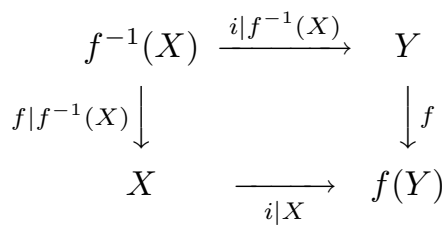

The partial mapping $f \mid f^{-1}(X): f^{-1}(X) \rightarrow X$ is obviously surjective and light. Furthermore, it is in $\mathcal{C}_{l c}$ as the restriction to the preimage of a closed subset of $Y$ of the $f \in \mathcal{C}_{l c}$. Moreover, the range space $X$ of this restriction is locally connected. Applying Proposition 11 we conclude that $f \mid f^{-1}(X)$ is open, whence, by implication $(\delta) \Longrightarrow\left(\omega_{0}\right)$ of Statement 1 (applied to the mapping $f \mid f^{-1}(X)$ ), there is a homeomorphic copy $X^{\prime}$ of $X$ in $f^{-1}(X)$ such that the (double) restriction $\left(f \mid f^{-1}(X)\right) \mid X^{\prime}: X^{\prime} \rightarrow X$ is a homeomorphism. Thus $\left(\omega_{0}(\mathcal{M})\right)$ is shown, and the proof is complete. 
14. Remark. In the Maćkowiak classification of mappings, locally confluent mappings, $\mathcal{L C}$, and semi-confluent ones, $\mathcal{S C}$, are the closest to the class $\mathcal{C}$ of confluent mappings; see [9, Table II, p. 28]. In Proposition 13, the implication $(\delta) \Longrightarrow\left(\omega_{0}(\mathcal{L C})\right)$ is shown. The implication $(\delta) \Longrightarrow\left(\omega_{0}(\mathcal{S C})\right)$ does not hold; see [9, Example 3.12, p. 14]. Consequently, the class of semi-confluent mappings cannot be attached to ones listed in Proposition 13.

In connection with Proposition 12, where local connectedness of either the domain or the range space of $f$ is assumed, consider the following variants of the previous conditions.

$\left(\lambda_{0}(\mathcal{M})\right)$ For every compact space $Y$, for every light mapping $f: Y \rightarrow f(Y)$ in $\mathcal{M}$ with locally connected $f(Y)$ and with $X \subset f(Y)$ and for every point $y_{0} \in f^{-1}(X) \subset Y$ there exists a homeomorphic copy $X^{\prime}$ of $X$ in $Y$ with $y_{0} \in X^{\prime}$ such that the restriction $f \mid X^{\prime}: X^{\prime} \rightarrow f\left(X^{\prime}\right)=X$ is a homeomorphism.

$(\lambda(\mathcal{M}))$ For every compact space $Y$ and for every light mapping $f: Y \rightarrow f(Y)$ in $\mathcal{M}$ with locally connected $f(Y)$ and with $X \subset f(Y)$ there exists a homeomorphic copy $X^{\prime}$ of $X$ in $Y$ such that the restriction $f \mid X^{\prime}: X^{\prime} \rightarrow$ $f\left(X^{\prime}\right)=X$ is a homeomorphism.

It is interesting to know whether the implication $(\lambda(\mathcal{M})) \Longrightarrow(\delta)$ holds for each continuum $X$ and any class $\mathcal{M}$ of mappings listed in (11). According to Proposition 12 it is enough to decide this for the class $\mathcal{O}$ of open mappings. We will show that this is indeed the case, i.e., that Theorem 9 of [1, p. 1842] can be strengthened in such a way that condition $(\omega)$ (equivalently $(\gamma)$, see Statement 1 ) is replaced by $(\lambda(\mathcal{O}))$.

15. Proposition. For each continuum $X$, the implication $(\lambda(\mathcal{O})) \Longrightarrow(\gamma)$ is true.

Proof. Let, as in $(\gamma)$, a continuum $Y$ and a light open mapping $f: Y \rightarrow f(Y)$ be given such that the continuum $X$ is a subset of $f(Y)$. By [11, Theorem 1, p. 175] there are locally connected continua $Y^{*}$ and $Z^{*}$ such that $Y$ and $f(Y)$ are subsets of $Y^{*}$ and $Z^{*}$, respectively, and there is an extension $f^{*}$ of $f$ from $Y^{*}$ onto $Z^{*}$ such that $f^{*}$ is open and

$$
f^{*}(Y) \cap f^{*}\left(Y^{*} \backslash Y\right)=\emptyset .
$$

According to the Whyburn factorization theorem for open mappings (see 15 . Theorem 4.2, p. 143]) $f^{*}$ can be uniquely factorized in the form $f^{*}=g \circ h$ so that $h: Y^{*} \rightarrow h\left(Y^{*}\right)$ is monotone, and $g: h\left(Y^{*}\right) \rightarrow Z^{*}$ is open and light. Since $X \subset f(Y) \subset Z^{*}$ and $Z^{*}$ is locally connected, applying the condition $(\lambda(\mathcal{O}))$ to the mapping $g$ we infer that there exists a continuum $X^{\prime \prime} \subset h\left(Y^{*}\right)$ that is homeomorphic to $X$ such that $g \mid X^{\prime \prime}: X^{\prime \prime} \rightarrow g\left(X^{\prime \prime}\right)=X$ is a homeomorphism. Furthermore, $h^{-1}\left(X^{\prime \prime}\right)=\left(f^{*}\right)^{-1}\left(g\left(X^{\prime \prime}\right)\right)=\left(f^{*}\right)^{-1}(X) \subset Y$ according to (15.1).

We claim that the partial mapping $h \mid Y: Y \rightarrow h(Y)$ is a homeomorphism. It is enough to show that it is one-to-one. So, take a point $w \in h(Y)$. If $h^{-1}(w) \cap$ $\left(Y^{*} \backslash Y\right) \neq \emptyset$, let $a \in Y$ and $b \in Y^{*} \backslash Y$ be points with $h(a)=w=h(b)$. Then $f^{*}(a)=g(h(a))=g(w)=g(h(b))=f^{*}(b)$, contrary to (15.1). Therefore $h^{-1}(w) \subset Y$. Since $h^{-1}(w)=\left(f^{*}\right)^{-1}(g(w))=f^{-1}(g(w))$ and since $f$ is light, we conclude that $h^{-1}(w)$ is a singleton. Thus $h \mid Y$ is a homeomorphism, as needed. 
Define $X^{\prime}=h^{-1}\left(X^{\prime \prime}\right) \subset Y$. Thus $X^{\prime}$ is a continuum that is homeomorphic with $X$, and $f\left|X^{\prime}=f^{*}\right| X^{\prime}=\left(g \mid h\left(X^{\prime}\right)\right) \circ\left(h \mid X^{\prime}\right)$ is a homeomorphism. Moreover, $f\left(X^{\prime}\right)=f^{*}\left(X^{\prime}\right)=g\left(h\left(X^{\prime}\right)\right)=g\left(X^{\prime \prime}\right)=X$. Therefore $(\gamma)$ is satisfied; so the proof is complete.

Corollary 10 of [1] and Proposition 13 imply, by Proposition 15, our main result.

16. Theorem. Let $\mathcal{M}$ denote any of the following classes of mappings: open, OM-mappings, confluent, locally confluent, and confluent over locally connected continua. Then for each continuum $X$, the conditions

$$
(\delta),\left(\gamma_{0}\right),(\gamma),\left(\omega_{0}(\mathcal{M})\right),(\omega(\mathcal{M})),\left(\lambda_{0}(\mathcal{M})\right) \text { and }(\lambda(\mathcal{M}))
$$

are equivalent.

We say that a continuum $X$ has the lifting property with respect to a class $\mathcal{M}$ of mappings between compact spaces provided that for every compact space $Y$, for every mapping $f: Y \rightarrow f(Y)$ in $\mathcal{M}$, for every mapping $g: X \rightarrow f(Y)$, for all points $x \in X$ and $y \in Y$ satisfying $g(x)=f(y)$, there is a mapping $\tilde{g}: X \rightarrow Y$ such that $\tilde{g}(x)=y$ and $g=f \circ \tilde{g}$.

In [12] Theorem 1, p. 41] it is proved that each dendrite $X$ has the lifting property with respect to light open mappings under an additional assumption that the mapping $g$ is onto. Actually, the surjectivity of $g$ is not restrictive; moreover, this result and Proposition 12 easily imply that each dendrite has the lifting property with respect to any class $\mathcal{M} \in\left\{\mathcal{O}, \mathcal{O} \mathcal{M}, \mathcal{C}, \mathcal{L C}, \mathcal{C}_{l c}\right\}$ of light mappings (see also [6]).

It follows from Theorem 16 that only dendrites have the property. Indeed, it is enough to check condition $\left(\omega\left(\mathcal{C}_{l c}\right)\right)$ for a continuum $X$ having the lifting property. So, take a compact space $Y$, a light mapping $f: Y \rightarrow f(Y)$ in $\mathcal{C}_{l c}$ such that $X \subset f(Y)$, a point $y_{0} \in f^{-1}(X) \subset Y$, and $x_{0}=f\left(y_{0}\right) \in X$. Let $g: X \rightarrow f(Y)$ be the inclusion mapping. By the lifting property there is a mapping $\tilde{g}: X \rightarrow Y$ with $\tilde{g}\left(x_{0}\right)=y_{0}$ and $g=f \circ \tilde{g}$. Take $X^{\prime}=\tilde{g}(X)$, and observe that $\tilde{g}: X \rightarrow X^{\prime}$ is a homeomorphism, and that $y_{0} \in X^{\prime}$. Hence $f \mid X^{\prime}: X^{\prime} \rightarrow f\left(X^{\prime}\right)=X$ is a homeomorphism, too.

Summarizing, we get the following corollary.

17. Corollary. A continuum $X$ has the lifting property with respect to a class $\mathcal{M} \in\left\{\mathcal{O}, \mathcal{O M}, \mathcal{C}, \mathcal{L C}, \mathcal{C}_{l c}\right\}$ of light mappings if and only if $X$ is a dendrite.

18. Remarks. (1) Corollary 17 is also true if, in the definition of the lifting property, we restrict compact spaces $Y$ to continua only (see Theorem 16 and condition $\left.\left(\gamma_{0}\right)\right)$.

(2) Conditions (16.1) are also equivalent for $\mathcal{M} \in\{\mathcal{O}, \mathcal{O M}, \mathcal{C}, \mathcal{L C}\}$ and an arbitrary compact Hausdorff (not necessarily metrizable) space $Y$, because in such settings the equivalences (12.1)-(12.4) and the implication $(\delta) \Longrightarrow(\omega(\mathcal{M}))$ hold true (see [12, Propositions 1 and 2, p. 39, and Corollary 1, p. 44]).

\section{REFERENCES}

1. J. J. Charatonik, W. J. Charatonik, and P. Krupski, Dendrites and light open mappings, Proc. Amer. Math. Soc. 128 (2000), 1839-1843. MR 2001c:54027

2. J. J. Charatonik, W. J. Charatonik, and S. Miklos, Confluent mappings of fans, Dissertationes Math. (Rozprawy Mat.) 301 (1990), 86 pp. MR 91h:54056

3. J. J. Charatonik and K. Omiljanowski, On light open mappings, Baku International Topological Conference Proceedings, ELM, Baku, 1989, pp. 211-219. 
4. R. Engelking and A. Lelek, Metrizability and weight of inverses under confluent mappings, Colloq. Math. 21 (1970), 239-246. MR 41:7646

5. W. T. Ingram, C-sets and mappings of continua, Topology Proc. 7 (1982), 83-90. MR 85i:54040

6. J. Krasinkiewicz, Path-lifting property for 0-dimensional confluent mappings, Bull. Polish Acad. Sci. Math. 48 (2000), 357-367. MR 2001h:54020

7. K. Kuratowski, Topology, vol. 2, Academic Press, New York, London and PWN Polish Scientific Publishers, Warsaw, 1968. MR 41:4467

8. A. Lelek and D. R. Read, Compositions of confluent mappings and some other classes of functions, Colloq. Math. 29 (1974), 101-112. MR 51:4142]

9. T. Maćkowiak, Continuous mappings on continua, Dissertationes Math. (Rozprawy Mat.) 158 (1979), 95 pp. MR 81a:54034

10. T. Maćkowiak, Singular arc-like continua, Dissertationes Math. (Rozprawy Mat.) 257 (1986), 40 pp. MR 88f:54066

11. T. Maćkowiak and E. D. Tymchatyn, Some properties of open and related mappings, Colloq. Math. 49 (1985), 175-194. MR 87g:54038

12. T. Maćkowiak and E. D. Tymchatyn, Some classes of locally connected continua, Colloq. Math. 52 (1987), 39-52. MR 88h:54047

13. J. Mioduszewski, Twierdzenie o selektorach funkcyj wielowartościowych na dendrytach $[A$ theorem on the selectors of multi-valued functions on dendrites], Prace Mat. 5 (1961), 73-77, in Polish; Russian and English summaries. MR 24:A534

14. S. B. Nadler, Jr., Continua determined by surjections of various types, preprint.

15. G. T. Whyburn, Analytic topology, American Mathematical Society Colloquium Publications, Vol. 28, Providence, RI, 1942, reprinted with corrections 1971. MR 4:86b

Instituto de Matemáticas, UnAm, Circuito Exterior, Ciudad Universitaria, 04510 MÉXico, D. F., MÉxico

E-mail address: jjc@matem.unam.mx

Mathematical Institute, University of WrocŁaw, Pl. Grunwaldzki 2/4, 50-384 WrocŁaW, Poland

E-mail address: krupski@math.uni.wroc.pl 